\title{
EXISTENCE PRINCIPLE FOR BVPS WITH STATE-DEPENDENT IMPULSES
}

\author{
IRENA RACHŮNKOVÁ - JAN TOMEČEK
}

\begin{abstract}
The paper provides an existence principle for the SturmLiouville boundary value problem with state-dependent impulses

$$
\begin{array}{cc}
z^{\prime \prime}(t)=f\left(t, z(t), z^{\prime}(t)\right) & \text { for a.e. } t \in[0, T] \subset \mathbb{R}, \\
z(0)-a z^{\prime}(0)=c_{1}, & z(T)+b z^{\prime}(T)=c_{2},
\end{array}
$$$$
z\left(\tau_{i}+\right)-z\left(\tau_{i}\right)=J_{i}\left(\tau_{i}, z\left(\tau_{i}\right)\right), \quad z^{\prime}\left(\tau_{i}+\right)-z^{\prime}\left(\tau_{i}-\right)=\mathcal{M}_{i}\left(\tau_{i}, z\left(\tau_{i}\right)\right),
$$

where the points $\tau_{1}, \ldots, \tau_{p}$ depend on $z$ through the equations

$$
\tau_{i}=\gamma\left(z\left(\tau_{i}\right)\right), \quad i=1, \ldots, p, p \in \mathbb{N} .
$$

Provided $a, b \in[0, \infty), c_{j} \in \mathbb{R}, j=1,2$, and the data functions $f, J_{i}$, $\mathcal{M}_{i}, i=1, \ldots, p$, are bounded, transversality conditions for barriers $\gamma_{i}$, $i=1, \ldots, p$, which yield the solvability of the problem, are delivered. An application to the problem with unbounded data functions is demonstrated.
\end{abstract}

\section{Introduction}

Impulsive differential equations have attracted lots of interest due to their important applications in many areas such as aircraft control, drug administration, and theshold theory in biology [4], [13], [20]. In practical ecological systems, the control measures are taken only when the amount of species reaches a threshold value, rather than the usual impulsive fixed-time control strategy. Studies of real

2010 Mathematics Subject Classification. 34B37, 34B15.

Key words and phrases. Impulsive differential equation, state-dependent impulses, SturmLiouville problem, second order ODE, transversality conditions.

Supported by grant IGA Mathematical models, PrF_2013_013. 
life problems with such state-dependent impulsive effects were made in [8], [11], [14], [15], [21], [22].

A very particular case of state-dependent impulses are impulses at fixed moments. This is the case that the moments, where impulses act in state variable, are known. The theory of these impulsive problems is widely developed and presents direct analogies with the methods and results for problems without impulses. Important texts in this area are [2], [3], [12], [19], [23].

A different situation arises, when the impulses appear in evolutionary trajectories fulfilling a predetermined relation between state and time variables. This case, which is represented by state-dependent impulses, is studied here. In particular, we investigate the solvability of boundary value problems with statedepending impulses. Such problems can be found for example in differential population models, where the densities of populations are subject to given conditions at the beginning and the end of the studied time interval and the impulses, caused by harvesting or fishering, act at the moments depending on the threshold values of the densities. The main reason that such problems are developed substantionally less than those with impulses at fixed moments is that new difficulties appear when examining state-dependent impulses. To demonstrate it, we compare these two types of impulses in the Dirichlet problem and show some fundamental differences between them.

1.1. Dirichlet problem with impulses at fixed moments. For $T \in$ $(0, \infty)$ and $A, B \in \mathbb{R}$, consider the Dirichlet problem

$$
\begin{aligned}
u^{\prime \prime}(t) & =f\left(t, u(t), u^{\prime}(t)\right), \quad \text { for a.e. } t \in[0, T], \\
u(0) & =A, \quad u(T)=B .
\end{aligned}
$$

Let a finite number of points $0=t_{0}<t_{1}<\ldots<t_{p}<t_{p+1}=T, p \in \mathbb{N}$, be given. We investigate the existence of a solution $u$ of problem (1.1), (1.2) subject to some impulse conditions at the moments $t_{1}, \ldots, t_{p}$.

For example, we choose impulse functions $J_{i}, \mathcal{M}_{i}, i=1, \ldots, p$, and define impulse conditions as

$$
\left\{\begin{array}{l}
u\left(t_{i}+\right)-u\left(t_{i}\right)=J_{i}\left(u\left(t_{i}\right)\right), \\
u^{\prime}\left(t_{i}+\right)-u^{\prime}\left(t_{i}-\right)=\mathcal{M}_{i}\left(u\left(t_{i}\right)\right),
\end{array} \quad i=1, \ldots, p .\right.
$$

We use the notation

$$
\lim _{t \rightarrow a+} z(t)=z(a+), \quad \lim _{t \rightarrow a-} z(t)=z(a-) .
$$

The impulsive problem (1.1)-(1.3) can be transformed to a fixed point problem in a suitable functional space as follows. Consider a set $X$ of functions $u:[0, T] \rightarrow \mathbb{R}$ 
defined by

$$
u(t)= \begin{cases}u_{[0]}(t) \quad \text { if } t \in\left[0, t_{1}\right], \\ u_{[1]}(t) \quad \text { if } t \in\left(t_{1}, t_{2}\right], \\ \ldots \ldots \ldots \ldots . \ldots . \cdots \\ u_{[p]}(t) \quad \text { if } t \in\left(t_{p}, T\right],\end{cases}
$$

where $u_{[i]} \in C^{1}\left(\left[t_{i}, t_{i+1}\right]\right), i=1, \ldots, p$. Then $X$ with the norm $\|u\|_{\infty}+\left\|u^{\prime}\right\|_{\infty}$, where $\|u\|_{\infty}=\operatorname{supess}_{t \in[0, T]}|u(t)|$, becomes a Banach space. It is known (see e.g. [16]) that a solution of problem (1.1)-(1.3) can be found as a fixed point of an operator $\mathcal{F}: X \rightarrow X$ which is given by

$$
\begin{aligned}
\mathcal{F} u(t)= & \int_{0}^{T} G(t, s) f\left(s, u(s), u^{\prime}(s)\right) d s+A+(B-A) \frac{t}{T} \\
& +\sum_{i=1}^{p} \frac{\partial G}{\partial s}\left(t, t_{i}\right) J_{i}\left(u\left(t_{i}\right)\right)+\sum_{i=1}^{p} G\left(t, t_{i}\right) \mathcal{M}_{i}\left(u\left(t_{i}\right)\right) .
\end{aligned}
$$

Here $G$ is the Green's function of the problem $u^{\prime \prime}(t)=0, u(0)=u(T)=0$. If $f$ fulfils the Carathéodory conditions on $[0, T] \times \mathbb{R}^{2}$ and $J_{i}, \mathcal{M}_{i}, i=1, \ldots, p$, are continuous on $\mathbb{R}$, then $\mathcal{F}$ is completely continuous. Therefore, having the Banach space $X$ and the operator $\mathcal{F}$ defined by (1.4), we can use similar conditions and arguments as for problem (1.1), (1.2) without impulses to get a fixed point of $\mathcal{F}$ in $X$.

1.2. Dirichlet problem with state-dependent impulses. Consider problem (1.1), (1.2) and choose a finite number of functions

$$
0<\gamma_{1}(x)<\gamma_{2}(x)<\ldots<\gamma_{p}(x)<T \text { for }|x| \leq K,
$$

where $K \in(0, \infty)$. Functions $\gamma_{i}$ will be called barriers here. We investigate the existence of a solution of problem (1.1), (1.2) subject to the impulse conditions

$$
\left\{\begin{array}{l}
u\left(\tau_{i}+\right)-u\left(\tau_{i}\right)=J_{i}\left(u\left(\tau_{i}\right)\right), \\
u^{\prime}\left(\tau_{i}+\right)-u^{\prime}\left(\tau_{i}-\right)=\mathcal{M}_{i}\left(u\left(\tau_{i}\right)\right),
\end{array} \quad i=1, \ldots, p,\right.
$$

where the points $\tau_{1}, \ldots, \tau_{p} \in(0, T)$ depend on $u$ through the equations

$$
\tau_{i}=\gamma_{i}\left(u\left(\tau_{i}\right)\right), \quad i=1, \ldots, p .
$$

Hence, $\tau_{i}$ are intersection points of graphs of $u$ with the barriers $\gamma_{i}, i=1, \ldots, p$.

1.3. Main differences between impulses at fixed moments and state-dependent impulses. We see that condition (1.3) contains $p$ points $t_{1}, \ldots, t_{p}$ which are given before and which are common for all solutions of problem (1.1)(1.3). In contrast to that, conditions (1.6) and (1.7) yield the following inconveniences. 
(i) Number of points $\tau_{i}$ given by (1.7). There are continuous functions $u$ and barriers $\gamma$ such that the equation

$$
\tau=\gamma(u(\tau))
$$

has infinitely many solutions $\tau_{u}$. On Figure 1 we see infinitely many intersection

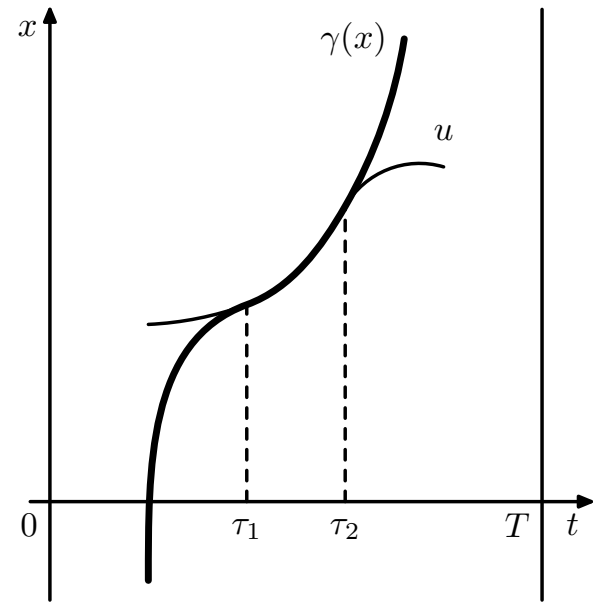

FiguRE 1. Infinitely many intersection points

points $\tau_{u}$ of $u$ with $\gamma$, which form an interval $\left[\tau_{1}, \tau_{2}\right]$. In this case $\mathcal{P}: u \mapsto \tau_{u}$ is a multivalued map.

(ii) Points $\tau_{i}$ need not depend on $u$ continuously. Consider functions in $C([0, T])$ having just one intersection point with $\gamma$. Figure 2 shows functions

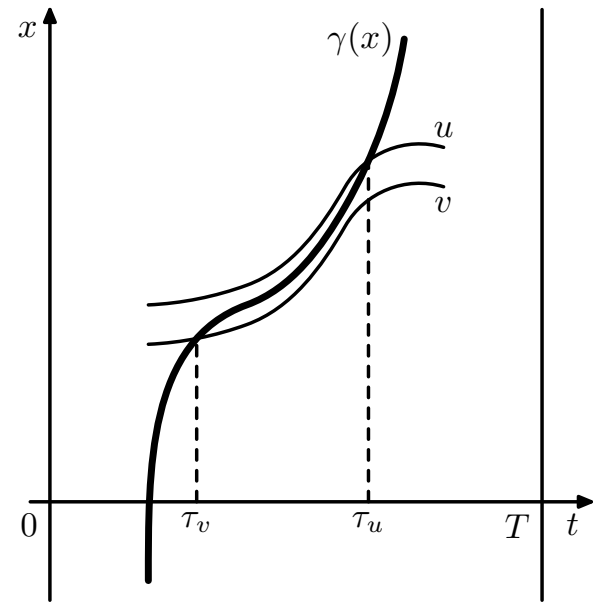

FiguRE 2. Intersection point $\tau_{u}$ does not depend on $u$ continuously 
$u$ and $v$ which are close to each other while their intersection points $\tau_{u}$ and $\tau_{v}$ are not. In this case an operator $\mathcal{P}: u \mapsto \tau_{u}$ can be defined on the set of such functions, but $\mathcal{P}$ is not continuous.

(iii) Beating of solutions. There are functions $f, \gamma, J, \mathcal{M}$ and constants $A, A_{1}$ such that a solution $u$ of equation (1.1) satisfying conditions

$$
\left\{\begin{array}{l}
u(0)=A, \quad u^{\prime}(0)=A_{1}, \\
u(\tau+)-u(\tau)=J(u(\tau)), \\
u^{\prime}(\tau+)-u^{\prime}(\tau-)=\mathcal{M}(u(\tau)), \quad \tau=\gamma(u(\tau)),
\end{array}\right.
$$

has a sequence of intersection points $\left\{\tau_{n}\right\}_{n=1}^{\infty}$ with the barrier $\gamma$ such that

$$
\lim _{n \rightarrow \infty} \tau_{n}=\tau^{*} \in(0, T)
$$

Hence, such a solution cannot be extended to $T$. This phenomenon, which is called beating, is presented on Figure 3. Here, $u$ is a solution of equation

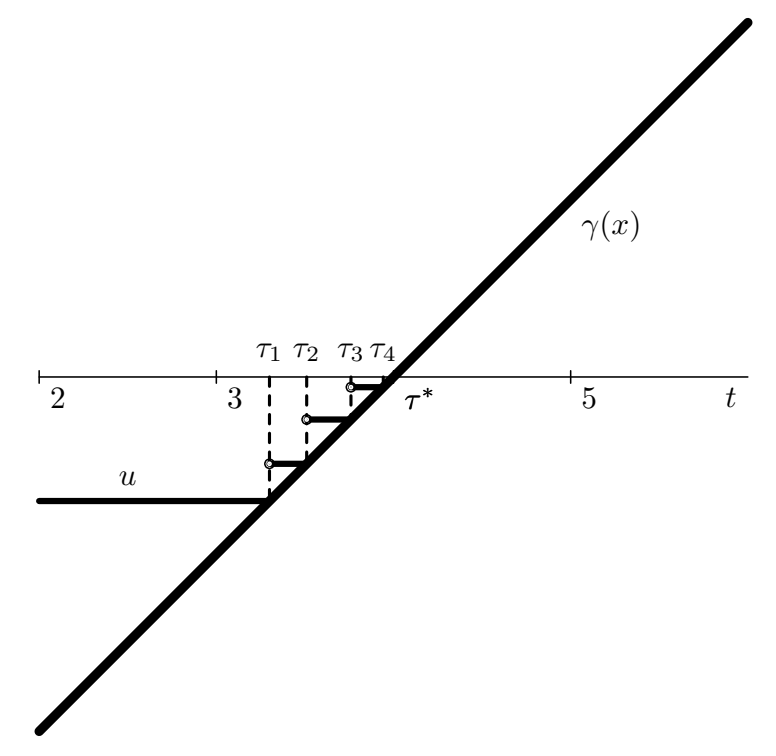

Figure 3. Beating of solution $u$

$u^{\prime \prime}(t)=0$ for $t \in[0, T]$ and satisfies conditions (1.8), where $A \in(-1,0), A_{1}=0$, $\mathcal{M} \equiv 0$ and

$$
J(x)=-x^{2}-x, \quad \gamma(x)=x+4 \quad \text { for }|x|<3 .
$$

We see that $\gamma$ fulfils (1.5) with $K=3$ provided $T>7$. Then $u$ is subject to an impulse effect at infinitely many moments $\tau_{n}$, and $\lim _{n \rightarrow \infty} \tau_{n}=\tau^{*}=4$, $\lim _{n \rightarrow \infty} u\left(\tau_{n}\right)=0$. Such solution cannot be extended to $T$. 
(iv) Fredholm property. A linear homogeneous problem corresponding to the impulse problem (1.1)-(1.3) has the form

$$
u^{\prime \prime}(t)=0, \quad u(0)=0, \quad u(T)=0,
$$

because for $J_{i}=\mathcal{M}_{i}=0, i=1, \ldots, p$, the impulses in (1.3) disappear. Since (1.9) has only the trivial solution, the Green's function of (1.9) exists. It is clear that for continuous and bounded functions $f, J_{i}, \mathcal{M}_{i}, i=1, \ldots, p$, and any $A$, $B \in \mathbb{R}$, the operator $\mathcal{F}$ from (1.4) has at least one fixed point, and hence problem (1.1)-(1.3) is solvable. The same is true if the continuity of $f$ is replaced by the Carathéodory conditions. This Fredholm property of problem (1.1)-(1.3) cannot be extended without some additional requirement to problem (1.1), (1.2), (1.6), (1.7). To demonstrate it, consider for simplicity

$$
\begin{gathered}
f \equiv 0, \quad A=-1, \quad B=0, \quad p=1, \quad J_{1}(x) \equiv 1, \quad \mathcal{M}_{1}(x) \equiv 1, \\
T \geq 10, \quad K=4, \quad \gamma_{1}(x)=5+x \quad \text { for }|x| \leq 4,
\end{gathered}
$$

that is problem $(1.1),(1.2),(1.6),(1.7)$ can be written as

$$
\left\{\begin{array}{l}
u^{\prime \prime}(t)=0, \quad u(0)=-1, \quad u(T)=0, \\
u(\tau+)-u(\tau)=1, \quad u^{\prime}(\tau+)-u^{\prime}(\tau-)=1, \\
\tau=5+u(\tau) \quad \text { for } \tau \in[1,9] .
\end{array}\right.
$$

Note that (1.9) is again a linear homogenous problem corresponding to (1.10). We observe that although $f, J_{1}$ and $\mathcal{M}_{1}$ in (1.10) are continuous and bounded functions, problem (1.10) is not solvable. It is obvious, because functions satisfying the equation $u^{\prime \prime}(t)=0$ on $[0, T]$ and the condition $u(0)=-1$ form the set $\{c t-1: c \in \mathbb{R}\}$.

- Let $c \in(-\infty,-3) \cup(5 / 9, \infty)$. Then the function $c t-1$ has no intersection point with $\gamma_{1}$ in $[1,9]$. But since $c T-1 \neq 0$, this function cannot be a solution of problem (1.10).

- Let $c \in[0,5 / 9]$. Then there is a unique intersection point $\tau_{1} \in[4,9]$ of the function $c t-1$ with $\gamma_{1}$ and there is no intersection point of the function $(1+c) t-\tau_{1}$ with $\gamma_{1}$ in $\left(\tau_{1}, 9\right]$. We see that the piece-wise linear function

$$
u(t)= \begin{cases}c t-1 & \text { if } t \in\left[0, \tau_{1}\right], \\ (1+c) t-\tau_{1} & \text { if } t \in\left(\tau_{1}, T\right],\end{cases}
$$

is subject to the impulse conditions of (1.10) but it does not vanish at $T$ and so it cannot be a solution of problem (1.10).

- Let $c \in[-3,0)$. Then we argue similarly and find that there are at most four points in $[1,9]$ at which the impulses occur. Denote the largest of them by $\tau^{*}$. If we construct a piece-wise linear function $u$ which is subject to the impulse conditions of (1.10), we get $u\left(\tau^{*}+\right)=\tau^{*}-4$ and 
$u^{\prime}(t) \geq 1$ for $t \in\left[\tau^{*}, T\right]$. Consequently $u$ does not vanish at $T$ and cannot be a solution of (1.10). See Figure 4 for $c=-3$.

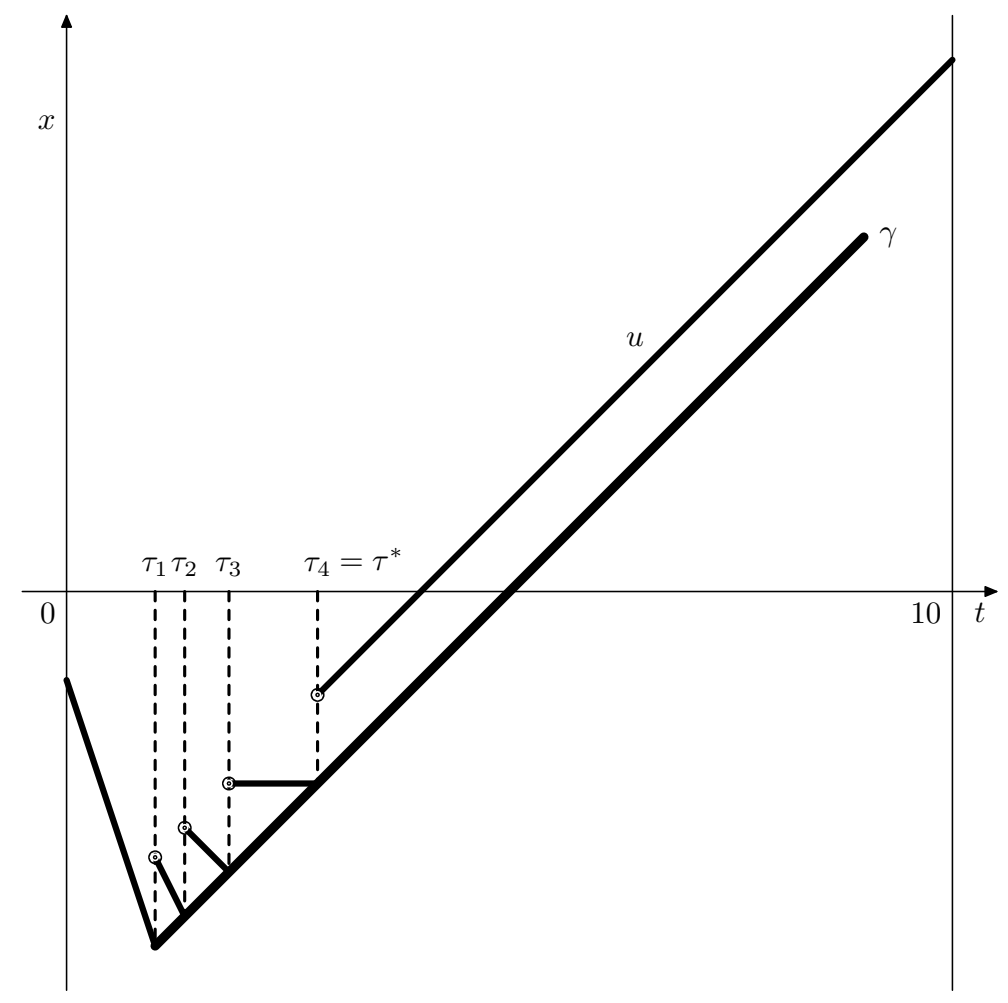

Figure 4. Solution $u$ for $c=-3$

Difficulties (i)-(iv) cause that if we search a transformation of problem (1.1), (1.2), (1.6), (1.7) to a fixed point problem of a suitable operator $\mathcal{F}$ in some functional space, a choice of this space is not a simple matter. Moreover, to define an operator $\mathcal{F}$ we cannot use a formula analogical to (1.4) substituing the intersection points $\tau_{i}$ instead of the given points $t_{i}$. It is because $\tau_{i}$ need not depend on $u$ continuously and consequently $\mathcal{F}$ defined by (1.4) with $\tau_{i}$ instead of $t_{i}$ need not be continuous.

This is the reason that almost all existence results for boundary value problems with state-dependent impulses have been reached for periodic problems. It is known that $n$-th order periodic problems can be transformed to fixed point problems of corresponding Poincaré maps in $\mathbb{R}^{n}$. Hence, the above difficulties with the construction of a functional space and an operator have been cleared in the periodic case. See e.g. [1], [5], [6], [9]. Other types of boundary value problems with state-dependent impulses have been studied very rarely. We managed to find the following papers. In the paper [10], the authors investigated the 
second order Sturm-Liouville problem through initial problems for multivalued maps. Their result is applicable to a quite special equation (1.1) where $f(t, x, y)$ vanishes on given regions in dependence on the values of $y$. The paper [7] deals with the first order differential inclusion subject to nonlinear boundary conditions. The papers [17] and [18] provide the existence results for the Dirichlet state-dependet impulsive problem.

Our main objective is to derive a general existence principle which will serve as a tool in the investigation of solvability of boundary value problems with statedependent impulses. To this aim we have delivered an approach which is substantially different from both citied papers and from papers dealing with impulses at fixed moments. We search neither a fixed point of multivalued map nor a fixed point of an operator in some space of discontinuous functions. Instead, considering $p$ barrier functions, we work in the $(p+1)$-th Cartesian power of the space $C^{1}([0, T])$. In more details, we define a convenient subset $\Omega \subset\left(C^{1}([0, T])\right)^{p+1}$. Then, to a given boundary value problem, we determine conditions for the barriers enabling to construct a completely continuous operator $\mathcal{F}: \bar{\Omega} \rightarrow \bar{\Omega}$ having a fixed point in $\bar{\Omega}$. A solution of the impulsive problem under consideration is then created from this fixed point. Here, this is done for the Sturm-Liouville boundary value problem, but we can proceed similarly in the case of other regular (and also singular) problems.

Our paper is organized as follows: In Section 2, we formulate the SturmLiouville BVP with state-dependent impulses and provide the transversality conditions for barriers $\gamma_{i}$. These conditions ensure unique transverse intersection of graphs of solutions with barriers. In Section 3 we prove the existence of a fixed point of an appropriate fixed point problem. Using this we state the existence principle for our BVP. Section 4 contains the existence result for the SturmLiouville BVP with state-dependent impulses and unbounded data functions $f$, $J_{i}, \mathcal{M}_{i}, i=1, \ldots, p$, whose proof is based on the existence principle of Section 3.

\section{Transversality conditions}

Let $T \in(0, \infty), p \in \mathbb{N}$. We investigate the following second order SturmLiouville boundary value problem on the interval $[0, T], T>0$, subject to $p$ state-dependent impulses

$$
\begin{aligned}
& z^{\prime \prime}(t)=f\left(t, z(t), z^{\prime}(t)\right) \quad \text { for a.e. } t \in[0, T], \\
& z(0)-a z^{\prime}(0)=c_{1}, \quad z(T)+b z^{\prime}(T)=c_{2}, \\
& \left\{\begin{array}{l}
z\left(\tau_{i}+\right)-z\left(\tau_{i}\right)=J_{i}\left(\tau_{i}, z\left(\tau_{i}\right)\right), \\
z^{\prime}\left(\tau_{i}+\right)-z^{\prime}\left(\tau_{i}-\right)=\mathcal{M}_{i}\left(\tau_{i}, z\left(\tau_{i}\right)\right), \\
\tau_{i}=\gamma_{i}\left(z\left(\tau_{i}\right)\right), \quad i=1, \ldots, p,
\end{array}\right.
\end{aligned}
$$


where

$$
\begin{gathered}
a, b \in[0, \infty), \quad c_{1}, c_{2} \in \mathbb{R}, \quad f \in \operatorname{Car}\left([0, T] \times \mathbb{R}^{2}\right), \\
J_{i}, \mathcal{M}_{i} \in C([0, T] \times \mathbb{R}), \quad \gamma_{i} \in C(\mathcal{D}), \quad \mathcal{D} \subset \mathbb{R}, \quad i=1, \ldots, p .
\end{gathered}
$$

Definition 2.1. A function $z:[0, T] \rightarrow \mathbb{R}$ is a solution of problem (2.1)(2.3), if for each $i \in\{1, \ldots, p\}$ there exists a unique $\tau_{i} \in(0, T)$ such that $\gamma_{i}\left(z\left(\tau_{i}\right)\right)=\tau_{i}, 0=\tau_{0}<\tau_{1}<\ldots<\tau_{p}<\tau_{p+1}=T$, the restrictions $\left.z\right|_{\left[\tau_{0}, \tau_{1}\right]}$, $\left.z\right|_{\left(\tau_{i}, \tau_{i+1}\right]}, i=1, \ldots, p$, have absolutely continuous derivatives, $z$ satisfies $(2.1)$ for almost every $t \in[0, T]$ and fulfils conditions (2.2) and (2.3).

Here, we denote by $C(J)$ the set of all continuous functions on the interval $J$, $C^{1}(J)$ the set of all functions having continuous derivatives on the interval $J$ and $L^{1}(J)$ the set of all Lebesgue integrable functions on $J$. For a compact interval $J$ we consider the linear spaces $C(J)$ and $C^{1}(J)$ equipped with the norms

$$
\|x\|_{\infty}=\max _{t \in J}|x(t)| \text { and }\|x\|^{*}=\|x\|_{\infty}+\left\|x^{\prime}\right\|_{\infty}
$$

respectively. In the paper we work with the linear space $X=\left(C^{1}([0, T])\right)^{p+1}$ equipped with the norm

$$
\left\|\left(u_{1}, \ldots, u_{p+1}\right)\right\|=\sum_{i=1}^{p+1}\left\|u_{i}\right\|^{*} \quad \text { for }\left(u_{1}, \ldots, u_{p+1}\right) \in X .
$$

It is well-known that the mentioned normed spaces are Banach spaces. Recall that for $\mathcal{A} \subset \mathbb{R}$, a function $f:[a, b] \times \mathcal{A} \rightarrow \mathbb{R}$ satisfies the Carathéodory conditions on $[a, b] \times \mathcal{A}$ (we write $f \in \operatorname{Car}([a, b] \times \mathcal{A}))$ if

- $f(\cdot, x):[a, b] \rightarrow \mathbb{R}$ is measurable for all $x \in \mathcal{A}$,

- $f(t, \cdot): \mathcal{A} \rightarrow \mathbb{R}$ is continuous for almost every $t \in[a, b]$,

- for each compact set $S \subset \mathcal{A}$ there exists a function $m_{S} \in L^{1}([a, b])$ such that $|f(t, x)| \leq m_{S}(t)$ for almost every $t \in[a, b]$ and each $x \in S$.

We assume that the data functions $f, J_{i}, \mathcal{M}_{i}$ are bounded, that is

$$
\left\{\begin{array}{l}
\text { there exist } m \in L^{1}([0, T]), A_{i}, B_{i} \in(0, \infty) \text { such that } \\
|f(t, x, y)| \leq m(t) \text { for almost every } t \in[0, T] \text { and all } x, y \in \mathbb{R} \\
\left|J_{i}(t, x)\right| \leq B_{i},\left|\mathcal{M}_{i}(t, x)\right| \leq A_{i} \text { for all } t \in[0, T], x \in \mathbb{R}, i=1, \ldots, p
\end{array}\right.
$$

In our approach we will exploit the Green's function of the linear homogenous BVP:

$$
\begin{gathered}
z^{\prime \prime}(t)=0, \quad t \in[0, T], \\
z(0)-a z^{\prime}(0)=0, \quad z(T)+b z^{\prime}(T)=0 .
\end{gathered}
$$


which has the form

$$
G(t, s)= \begin{cases}g(t, s) & \text { for } 0 \leq t \leq s \leq T \\ g(s, t) & \text { for } 0 \leq s \leq t \leq T\end{cases}
$$

where

$$
g(t, s)=\frac{(a+t)(b+T-s)}{T+a+b}, \quad t, s \in[0, T] .
$$

Further put

$$
g_{1}(t)=\frac{b+T-t}{T+a+b}, \quad g_{2}(t)=\frac{-a-t}{T+a+b}, \quad t \in[0, T]
$$

and denote a solution of problem (2.7), (2.2) by $\ell$. Evidently, there exist positive constants $C_{0}, C_{1}, C_{2}, L, L_{1}$ such that for $s, t \in[0, T]$ it holds

$$
\begin{gathered}
|g(t, s)| \leq C_{0}, \quad\left|g_{i}(t)\right| \leq C_{1}, \quad\left|g_{i}^{\prime}(t)\right| \leq C_{2}, \quad i=1,2, \\
|\ell(t)| \leq L, \quad\left|\ell^{\prime}(t)\right| \leq L_{1} .
\end{gathered}
$$

Finally, denote

$$
\left\{\begin{array}{l}
M=\int_{0}^{T} m(t) d t \\
K=C_{0} M+L+C_{0} \sum_{i=1}^{p} A_{i}+C_{1} \sum_{i=1}^{p} B_{i} \\
K_{1}=C_{1} M+L_{1}+C_{1} \sum_{i=1}^{p} A_{i}+C_{2} \sum_{i=1}^{p} B_{i} .
\end{array}\right.
$$

Now, we are ready to state the following transversality conditions:

$$
0<\min _{[-K, K]} \gamma_{1}<\max _{[-K, K]} \gamma_{i-1}<\min [-K, K] \gamma_{i}<\max _{[-K, K]} \gamma_{p}<b,
$$

for $i=2, \ldots, p$,

$$
\gamma_{i} \in C^{1}([-K, K]), \quad\left|\gamma_{i}^{\prime}(x)\right|<\frac{1}{K_{1}},
$$

$x \in[-K, K], i=1, \ldots, p$, and

$$
\begin{cases}\text { for each } i \in\{1, \ldots, p\} \text { either } & \\ J_{i}(t, x)=0 & t \in[0, T], x \in[-K, K], \text { or } \\ \gamma_{i}^{\prime}(x) \geq 0 \text { and } \quad J_{i}(t, x) \leq 0, & t \in[0, T], x \in[-K, K], \text { or } \\ \gamma_{i}^{\prime}(x) \leq 0 \quad \text { and } \quad J_{i}(t, x) \geq 0, & t \in[0, T], x \in[-K, K] .\end{cases}
$$

Define a set $\mathcal{B}$ by

$$
\mathcal{B}=\left\{u \in C^{1}([0, T]):\|u\|_{\infty}<K,\left\|u^{\prime}\right\|_{\infty}<K_{1}\right\} .
$$

The next lemma states that functions of $\overline{\mathcal{B}}$ have a unique trasverse intersection with each barrier. 
Lemma 2.2. Let $u \in \overline{\mathcal{B}}, i \in\{1, \ldots, p\}$ and let $\gamma_{i}$ satisfy (2.15)-(2.16). Then there exists a unique $\tau_{i} \in(0, T)$ such that

$$
\gamma_{i}\left(u\left(\tau_{i}\right)\right)=\tau_{i}
$$

Proof. Let us take an arbitrary $u \in \overline{\mathcal{B}}$ and $i \in\{1, \ldots, p\}$. Obviously, the constant $\tau_{i}$ is a solution of the equation $\gamma_{i}(u(t))=t$, i.e. $\tau_{i}$ is a root of the function $\sigma(t)=\gamma_{i}(u(t))-t, t \in[0, T]$. According to (2.15)-(2.16) and (2.18), we get $\sigma(0)=\gamma_{i}(u(0))>0, \sigma(T)=\gamma_{i}(u(T))-T<0$ and

$$
\sigma^{\prime}(t)=\gamma_{i}^{\prime}(u(t)) u^{\prime}(t)-1 \leq\left|\gamma_{i}^{\prime}(u(t))\right|\left|u^{\prime}(t)\right|-1<\frac{1}{K_{1}} K_{1}-1=0,
$$

for $t \in(0, T)$. Therefore $\sigma$ is strictly decreasing on $[0, T]$ and hence it has exactly one root in $(0, T)$.

Due to Lemma 2.2 we can define functionals $\mathcal{P}_{i}: \overline{\mathcal{B}} \rightarrow(0, T)$ by $\mathcal{P}_{i} u=\tau_{i}$, where $\tau_{i}$ fulfils $(2.19)$ for $i=1, \ldots, p$. Now, we will prove their continuity.

Lemma 2.3. Let $i \in\{1, \ldots, p\}$ and let $\gamma_{i}$ satisfy (2.15)-eqred11a. Then the functional $\mathcal{P}_{i}$ is continuous on $\overline{\mathcal{B}}$.

Proof. Let us consider $u_{n}, u \in \overline{\mathcal{B}}$ for $n \in \mathbb{N}$ such that $u_{n} \rightarrow u$ in $C^{1}([0, T])$. Choose $i \in\{1, \ldots, p\}$ and denote

$$
\sigma_{n}(t)=\gamma_{i}\left(u_{n}(t)\right)-t, \quad \sigma(t)=\gamma_{i}(u(t))-t, \quad \text { for } t \in[0, T], n \in \mathbb{N} .
$$

By Lemma 2.2, $\sigma_{n}\left(\tau_{i}^{n}\right)=0$ and $\sigma\left(\tau_{i}\right)=0$, where $\tau_{i}^{n}=\mathcal{P}_{i} u_{n}$ and $\tau_{i}=\mathcal{P}_{i} u$, respectively. According to $(2.15)-(2.16)$ we get $\sigma_{n}, \sigma \in C^{1}([0, T])$ for $n \in \mathbb{N}$ and

$$
\sigma_{n} \rightarrow \sigma \quad \text { in } C([0, T])
$$

We will prove that $\lim _{n \rightarrow \infty} \tau_{i}^{n}=\tau_{i}$. Let us take an arbitrary $\varepsilon>0$. Since $\sigma\left(\tau_{i}\right)=0$ and $\sigma^{\prime}\left(\tau_{i}\right)<0$ (cf. $\left.(2.20)\right)$, we can find $\xi \in\left(\tau_{i}-\varepsilon, \tau_{i}\right)$ and $\eta \in\left(\tau_{i}, \tau_{i}+\varepsilon\right)$ such that $\sigma(\xi)>0$ and $\sigma(\eta)<0$. From (2.21) it follows the existence of $n_{0} \in \mathbb{N}$ such that $\sigma_{n}(\xi)>0$ and $\sigma_{n}(\eta)<0$ for each $n \geq n_{0}$. By Lemma 2.2 and the continuity of $\sigma_{n}$ it follows that $\tau_{i}^{n} \in(\xi, \eta) \subset\left(\tau_{i}-\varepsilon, \tau_{i}+\varepsilon\right)$ for $n \geq n_{0}$.

\section{Existence principle}

In this section, in order to obtain the existence principle to problem (2.1)(2.3), we assume that conditions (2.4), (2.6), (2.15), (2.16) and (2.17) with $K$ and $K_{1}$ by (2.14) are fulfilled. Having the set $\mathcal{B}$ of (2.18), we construct a fixed point problem in the set $\bar{\Omega}$, where

$$
\Omega=\mathcal{B}^{p+1} \subset X
$$


For this purpose we define a functional $f_{u}$ as follows. Let $\mathcal{P}_{i}, i=1, \ldots, p$, be the functionals of Lemma 2.3. We set for almost every $t \in[0, T]$ and for each $u=\left(u_{1}, \ldots, u_{p+1}\right) \in \bar{\Omega}$

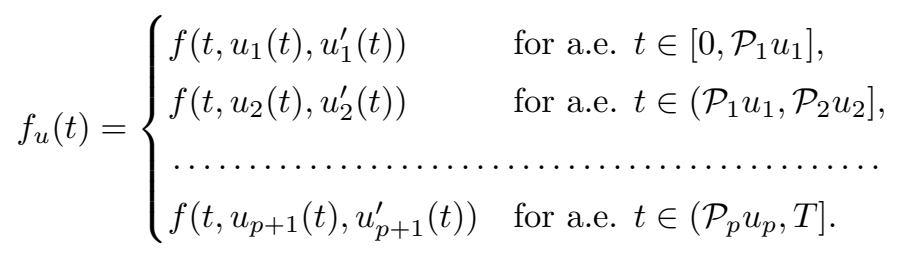

Note that for each $u_{i} \in \overline{\mathcal{B}}$ the point $\mathcal{P}_{i} u_{i} \in(0, T)$ is uniquely determined. Now, we can define an operator $\mathcal{F}: \bar{\Omega} \rightarrow X$ by $\mathcal{F}\left(u_{1}, \ldots, u_{p+1}\right)=\left(x_{1}, \ldots, x_{p+1}\right)$, where

$$
\begin{aligned}
x_{j}(t)= & \int_{0}^{T} G(t, s) f_{u}(s) d s+\ell(t) \\
& +\sum_{j \leq i \leq p}\left[-g\left(t, \mathcal{P}_{i} u_{i}\right) \mathcal{M}_{i}\left(\mathcal{P}_{i} u_{i}, u_{i}\left(\mathcal{P}_{i} u_{i}\right)\right)+g_{2}(t) J_{i}\left(\mathcal{P}_{i} u_{i}, u_{i}\left(\mathcal{P}_{i} u_{i}\right)\right)\right] \\
& +\sum_{1 \leq i<j}\left[-g\left(\mathcal{P}_{i} u_{i}, t\right) \mathcal{M}_{i}\left(\mathcal{P}_{i} u_{i}, u_{i}\left(\mathcal{P}_{i} u_{i}\right)\right)+g_{1}(t) J_{i}\left(\mathcal{P}_{i} u_{i}, u_{i}\left(\mathcal{P}_{i} u_{i}\right)\right)\right]
\end{aligned}
$$

for $t \in[0, T], j=1, \ldots, p+1$. Here, $G, g_{1}$ and $g_{2}$ are from (2.9), (2.10) and (2.11) and $\ell$ is a solution of problem (2.7), (2.2).

LEMma 3.1. The operator $\mathcal{F}$ has a fixed point in $\bar{\Omega}$.

Proof. According to (2.4) and (3.2), the operator $\mathcal{H}: \bar{\Omega} \rightarrow C^{1}([0, T])$, $(\mathcal{H} u)(t)=\int_{0}^{T} G(t, s) f_{u}(s) d s$ is compact on $\bar{\Omega}$. Since $J_{i}, \mathcal{M}_{i}$ are continuous on $[0, T] \times \mathbb{R}$ for $i=1, \ldots, p$ and $\mathcal{P}_{i}, i=1, \ldots, p$, are continuous on $\overline{\mathcal{B}}$ due to Lemma 2.3, we get by the Lebesgue dominated convergence theorem and the Arzelà-Ascoli theorem that $\mathcal{F}$ is compact on $\bar{\Omega}$. By (2.6), (2.12)-(2.14) and (3.2), we get from (3.3)

$$
\left|x_{j}(t)\right| \leq C_{0} M+L+C_{0} \sum_{i=1}^{p} A_{i}+C_{1} \sum_{i=1}^{p} B_{i}=K \quad \text { for } t \in[0, T],
$$

$j=1, \ldots, p+1$. Differentiating (3.3), we get

$$
\begin{aligned}
x_{j}^{\prime}(t)= & \int_{0}^{T} \frac{\partial G}{\partial t}(t, s) f_{u}(s) d s+\ell^{\prime}(t) \\
& +\sum_{j \leq i \leq p}\left[-g_{1}\left(\mathcal{P}_{i} u_{i}\right) \mathcal{M}_{i}\left(\mathcal{P}_{i} u_{i}, u_{i}\left(\mathcal{P}_{i} u_{i}\right)\right)+g_{2}^{\prime}(t) J_{i}\left(\mathcal{P}_{i} u_{i}, u_{i}\left(\mathcal{P}_{i} u_{i}\right)\right)\right] \\
& +\sum_{1 \leq i<j}\left[-g_{2}\left(\mathcal{P}_{i} u_{i}\right) \mathcal{M}_{i}\left(\mathcal{P}_{i} u_{i}, u_{i}\left(\mathcal{P}_{i} u_{i}\right)\right)+g_{1}^{\prime}(t) J_{i}\left(\mathcal{P}_{i} u_{i}, u_{i}\left(\mathcal{P}_{i} u_{i}\right)\right)\right]
\end{aligned}
$$

for $t \in[0, T], j=1, \ldots, p+1$. This yields similarly as before

$$
\left|x_{j}^{\prime}(t)\right| \leq C_{1} M+L_{1}+C_{1} \sum_{i=1}^{p} A_{i}+C_{2} \sum_{i=1}^{p} B_{i}=K_{1} \quad \text { for } t \in[0, T],
$$


$j=1, \ldots, p+1$. Therefore $x_{j} \in \overline{\mathcal{B}}$ for $j=1, \ldots, p+1$, and so $\left(x_{1}, \ldots, x_{p+1}\right) \in \bar{\Omega}$. Consequently $\mathcal{F}(\bar{\Omega}) \subset \bar{\Omega}$ and the Schauder fixed point theorem yields a fixed point in $\bar{\Omega}$.

The main result of this section is contained in the next theorem.

THEOREM 3.2 (Existence principle for problem (2.1)-(2.3)). Let assumptions (2.4), (2.6), (2.15), (2.16) and (2.17) with $K$ and $K_{1}$ by (2.14) be fulfilled. Then there exists a solution $z$ of problem (2.1)-(2.3) such that

$$
\sup _{t \in[0, T]}|z(t)| \leq K, \quad \sup _{t \in[0, T]}\left|z^{\prime}(t)\right| \leq K_{1} .
$$

Proof. By Lemma 3.1, there exists $u=\left(u_{1}, \ldots, u_{p+1}\right) \in \bar{\Omega}$, which is a fixed point of the operator $\mathcal{F}$ defined in (3.3). This means that

$$
\begin{aligned}
u_{j}(t)= & \int_{0}^{T} G(t, s) f_{u}(s) d s+\ell(t) \\
& +\sum_{j \leq i \leq p}\left[-g\left(t, \mathcal{P}_{i} u_{i}\right) \mathcal{M}_{i}\left(\mathcal{P}_{i} u_{i}, u_{i}\left(\mathcal{P}_{i} u_{i}\right)\right)+g_{2}(t) J_{i}\left(\mathcal{P}_{i} u_{i}, u_{i}\left(\mathcal{P}_{i} u_{i}\right)\right)\right] \\
& +\sum_{1 \leq i<j}\left[-g\left(\mathcal{P}_{i} u_{i}, t\right) \mathcal{M}_{i}\left(\mathcal{P}_{i} u_{i}, u_{i}\left(\mathcal{P}_{i} u_{i}\right)\right)+g_{1}(t) J_{i}\left(\mathcal{P}_{i} u_{i}, u_{i}\left(\mathcal{P}_{i} u_{i}\right)\right)\right]
\end{aligned}
$$

for $t \in[0, T], j=1, \ldots, p+1$ and

$$
\begin{aligned}
u_{j}^{\prime}(t)= & \int_{0}^{T} \frac{\partial G}{\partial t}(t, s) f_{u}(s) d s+\ell^{\prime}(t) \\
& +\sum_{j \leq i \leq p}\left[-g_{1}\left(\mathcal{P}_{i} u_{i}\right) \mathcal{M}_{i}\left(\mathcal{P}_{i} u_{i}, u_{i}\left(\mathcal{P}_{i} u_{i}\right)\right)+g_{2}^{\prime}(t) J_{i}\left(\mathcal{P}_{i} u_{i}, u_{i}\left(\mathcal{P}_{i} u_{i}\right)\right)\right] \\
& +\sum_{1 \leq i<j}\left[-g_{2}\left(\mathcal{P}_{i} u_{i}\right) \mathcal{M}_{i}\left(\mathcal{P}_{i} u_{i}, u_{i}\left(\mathcal{P}_{i} u_{i}\right)\right)+g_{1}^{\prime}(t) J_{i}\left(\mathcal{P}_{i} u_{i}, u_{i}\left(\mathcal{P}_{i} u_{i}\right)\right)\right]
\end{aligned}
$$

for $t \in[0, T], j=1, \ldots, p+1$. Now, for $t \in[0, T]$ define a function $z$ by

$$
z(t)=\left\{\begin{array}{l}
u_{1}(t) \quad t \in\left[0, \mathcal{P}_{1} u_{1}\right], \\
u_{2}(t) \quad t \in\left(\mathcal{P}_{1} u_{1}, \mathcal{P}_{2} u_{2}\right], \\
\ldots \ldots \ldots \ldots \ldots . . . \\
u_{p+1}(t) \quad t \in\left(\mathcal{P}_{p} u_{p}, T\right],
\end{array}\right.
$$

and denote

$$
\mathcal{P}_{j} u_{j}=\tau_{j}, \quad j=1, \ldots, p, \quad \tau_{0}=0, \quad \tau_{p+1}=T .
$$


Having in mind that $\ell$ fulfils (2.2), we get due to (2.9)-(2.11), (3.5) and (3.6),

$$
\begin{aligned}
z(0)-a z^{\prime}(0)= & u_{1}(0)-a u_{1}^{\prime}(0) \\
= & \int_{0}^{T}\left(G(0, s)-a \frac{\partial G}{\partial t}(0, s)\right) f_{u}(s) d s+\ell(0)-a \ell^{\prime}(0) \\
& +\sum_{1 \leq i \leq p}\left[-\left(g\left(0, \tau_{i}\right)-a g_{1}\left(\tau_{i}\right)\right) \mathcal{M}_{i}\left(\tau_{i}, u_{i}\left(\tau_{i}\right)\right)\right. \\
& \left.+\left(g_{2}(0)-a g_{2}^{\prime}(0)\right) J_{i}\left(\tau_{i}, u_{i}\left(\tau_{i}\right)\right)\right]=c_{1}, \\
z(T)+b z^{\prime}(T)= & u_{p+1}(T)+b u_{p+1}^{\prime}(T) \\
= & \int_{0}^{T}\left(G(T, s)+b \frac{\partial G}{\partial t}(T, s)\right) f_{u}(s) d s+\ell(T)+b \ell^{\prime}(T) \\
& +\sum_{1 \leq i<p+1}\left[-\left(g\left(\tau_{i}, T\right)+b g_{2}\left(\tau_{i}\right)\right) \mathcal{M}_{i}\left(\tau_{i}, u_{i}\left(\tau_{i}\right)\right)\right. \\
& \left.+\left(g_{1}(T)+b g_{1}^{\prime}(T)\right) J_{i}\left(\tau_{i}, u_{i}\left(\tau_{i}\right)\right)\right]=c_{2} .
\end{aligned}
$$

We have proved that $z$ fulfils (2.2). By Lemma $2.2, \tau_{j}$ is a unique point in $(0, T)$ satisfying

$$
\tau_{j}=\gamma_{j}\left(u_{j}\left(\tau_{j}\right)\right), \quad j=1, \ldots, p .
$$

Choose $j \in\{1, \ldots, p\}$. In view of (3.5) and (3.7) we get

$$
\begin{aligned}
z\left(\tau_{j}+\right)-z\left(\tau_{j}\right) & =u_{j+1}\left(\tau_{j}\right)-u_{j}\left(\tau_{j}\right) \\
& =\left(g_{1}\left(\tau_{j}\right)-g_{2}\left(\tau_{j}\right)\right) J_{j}\left(\tau_{j}, u_{j}\left(\tau_{j}\right)\right)=J_{j}\left(\tau_{j}, u_{j}\left(\tau_{j}\right)\right)=J_{j}\left(\tau_{j}, z\left(\tau_{j}\right)\right),
\end{aligned}
$$

and (3.6), (3.7) provide

$$
\begin{aligned}
z^{\prime}\left(\tau_{j}+\right)-z^{\prime}\left(\tau_{j}-\right) & =u_{j+1}^{\prime}\left(\tau_{j}\right)-u_{j}^{\prime}\left(\tau_{j}\right)=-\left(g_{2}\left(\tau_{j}\right)-g_{1}\left(\tau_{j}\right)\right) \mathcal{M}_{j}\left(\tau_{j}, u_{j}\left(\tau_{j}\right)\right) \\
& =\mathcal{M}_{j}\left(\tau_{j}, u_{j}\left(\tau_{j}\right)\right)=\mathcal{M}_{j}\left(\tau_{j}, z\left(\tau_{j}\right)\right) .
\end{aligned}
$$

We see that $z$ satisfies (2.3). The first condition in (2.15)-(2.16) yields $0<\tau_{1}<$ $\tau_{2}<\ldots<\tau_{p}<T$. Further we get from (3.2), (3.6)-(3.8)

$$
z^{\prime \prime}(t)=u_{j}^{\prime \prime}(t)=f_{u}(t)=f\left(t, u_{j}(t), u_{j}^{\prime}(t)\right)=f\left(t, z(t), z^{\prime}(t)\right)
$$

for almost every $t \in\left(\tau_{j-1}, \tau_{j}\right), j=1, \ldots, p+1$. We get that $z$ is a solution of equation (2.1). Finally, we will show that $\tau_{j}, j=1, \ldots, p$, are unique solutions of equations

$$
\tau_{j}=\gamma_{j}\left(z\left(\tau_{j}\right)\right), \quad j=1, \ldots, p .
$$

For this purpose it suffices to prove

$$
t \neq \gamma_{j}\left(u_{j+1}(t)\right), \quad t \in\left(\tau_{j}, T\right], j=1, \ldots, p .
$$

Choose $j \in\{1, \ldots, p\}$. By Lemma 3.1 and (3.1), $u_{j+1} \in \overline{\mathcal{B}}$ (see (2.18)) and hence $\left\|u_{j+1}\right\|_{\infty} \leq K$. According to assumption (2.17) there are three possibilities: 
(i) Assume that the first condition in (2.17) is satisfied. Then $J_{j}\left(\tau_{j}, x\right)=0$ for $x \in[-K, K]$ and we get by (2.3) and (3.7)

$$
u_{j+1}\left(\tau_{j}\right)-u_{j}\left(\tau_{j}\right)=z\left(\tau_{j}+\right)-z\left(\tau_{j}\right)=0 .
$$

Hence $\tau_{j}$ is a solution of the equation

$$
t=\gamma_{j}\left(u_{j+1}(t)\right) .
$$

By Lemma 2.2, equation (3.11) has a unique solution in $(0, T)$, which implies (3.10).

(ii) Assume that the second condition in (2.17) is satisfied. Then $\gamma_{j}^{\prime}(x) \geq 0$ and $J_{j}\left(\tau_{j}, x\right) \leq 0$ for $x \in[-K, K]$. Put $\sigma(t)=\gamma_{j}\left(u_{j+1}(t)\right)-t$ for $t \in[0, T]$. It follows from (2.3) and (3.7) that

$$
u_{j+1}\left(\tau_{j}\right)-u_{j}\left(\tau_{j}\right)=z\left(\tau_{j}+\right)-z\left(\tau_{j}\right)=J_{j}\left(\tau_{j}, z\left(\tau_{j}\right)\right) \leq 0
$$

and

$$
\sigma\left(\tau_{j}\right)=\gamma_{j}\left(u_{j+1}\left(\tau_{j}\right)\right)-\tau_{j} \leq \gamma_{j}\left(u_{j}\left(\tau_{j}\right)\right)-\tau_{j}=0
$$

due to (3.9). The second condition in (2.15)-(2.16) gives

$$
\sigma^{\prime}(t)=\gamma_{j}^{\prime}\left(u_{j+1}(t)\right) u_{j+1}^{\prime}(t)-1<\frac{1}{K_{1}} K_{1}-1=0
$$

for $t \in\left(\tau_{j}, T\right)$. So, (3.10) is valid.

(iii) The third condtion in (2.17) is dual to the second one, so the proof is similar.

\section{Unbounded data functions}

Assume that condition (2.6) fails, that is at least one of the data functions $f$, $J_{i}, \mathcal{M}_{i}, i=1, \ldots, p$, in problem (2.1)-(2.3) is unbounded. Then the constants $K$ and $K_{1}$, which are needed in the transversality conditions (2.15)-(2.16) and (2.17), cannot be obtained by (2.14). The next lemma gives constants $K$ and $K_{1}$ which will serve as a priori estimates of solutions of problem (2.1)-(2.3) and which can be used in (2.15), (2.16) and (2.17), provided $f(t, \cdot, \cdot), J_{i}(t, \cdot)$ and $\mathcal{M}_{i}(t, \cdot), i=1, \ldots, p$, have at most sublinear growth in large values of their space variables (see Theorem 4.2).

Lemma 4.1 (A priori estimates). Consider condition (2.2), where $a, b \in$ $[0, \infty), c_{1}, c_{2} \in \mathbb{R}$. Let $C_{0}, C_{1}, C_{2}$ and $L, L_{1}$ be constants satisfying (2.12) and (2.13), respectively. For $\mathcal{D}=[0, \infty) \times[0, \infty)$, assume that $\tilde{f} \in \operatorname{Car}([0, T] \times \mathcal{D})$ is nondecreasing in its second and third variable for almost every $t \in[0, T]$, and $\widetilde{J}_{i}, \widetilde{\mathcal{M}}_{i} \in C[0, \infty)$ are nondecreasing for $i=1, \ldots, p$. Finally, let

$$
\lim _{x \rightarrow \infty} \frac{1}{x} \int_{0}^{T} \widetilde{f}(t, x, x) d t+\widetilde{J}_{i}(x)+\widetilde{\mathcal{M}}_{i}(x)=0, \quad i=1, \ldots, p .
$$


Then there exists $K^{*}>0$ such that each $K_{1} \in\left(K^{*}, \infty\right)$ satisfies

$$
K_{1}>C_{1} \int_{0}^{T} \widetilde{f}\left(t, K, K_{1}\right) d t+L_{1}+C_{1} \sum_{i=1}^{p} \widetilde{\mathcal{M}}_{i}(K)+C_{2} \sum_{i=1}^{p} \widetilde{J}_{i}(K),
$$

where $K>K_{1}$ is a solution of the equation

$$
K=\alpha K_{1}+\left|c_{1}\right|+\sum_{i=1}^{p} \widetilde{J}_{i}(K)
$$

and

$$
\alpha=\max \{a+(p+1) T, 1\} .
$$

Proof. First, we will show that for each $K_{1}>0$ equation (4.3) has at least one solution $K>K_{1}$. Choose $K_{1}>0$. We see that the function

$$
\Phi(K)=\alpha K_{1}+\left|c_{1}\right|+\sum_{i=1}^{p} \widetilde{J}_{i}(K)
$$

is continuous on $[0, \infty)$ and $\Phi\left(K_{1}\right)-K_{1}>0$.

On the other hand, since $\lim _{K \rightarrow \infty} \Phi(K) / K=0$ due to (4.1), it holds $\Phi(K)-K<$ 0 for large $K$. Hence there exists at least one $K>K_{1}$ such that $\Phi(K)-K=0$.

Now, assume on the contrary, that for any $K_{1} \in(0, \infty)$ it holds

$$
K_{1} \leq C_{1} \int_{0}^{T} \widetilde{f}(t, K, K) d t+L_{1}+C_{1} \sum_{i=1}^{p} \widetilde{M}_{i}(K)+C_{2} \sum_{i=1}^{p} \widetilde{J}_{i}(K),
$$

where $K>K_{1}$ is a solution of (4.3). Then (4.3)-(4.5) give

$$
\begin{aligned}
& 1=\frac{\alpha K_{1}+\left|c_{1}\right|+\sum_{i=1}^{p} \widetilde{J}_{i}(K)}{K} \leq \alpha\left[\frac{C_{1}}{K} \int_{0}^{T} \widetilde{f}(t, K, K) d t+\frac{L_{1}}{K}\right. \\
& \left.\quad+C_{1} \sum_{i=1}^{p} \frac{\widetilde{M}_{i}(K)}{K}+C_{2} \sum_{i=1}^{p} \frac{\widetilde{J}_{i}(K)}{K}\right]+\frac{\left|c_{1}\right|}{K}+\sum_{i=1}^{p} \frac{\widetilde{J}_{i}(K)}{K} .
\end{aligned}
$$

Letting $K_{1} \rightarrow \infty$ we get $K \rightarrow \infty$ and, due to (4.1), the contradiction $1 \leq 0$ follows.

We are ready to formulate the main result of this section.

THEOREM 4.2. Let (2.4) hold and let us assume that there exist functions $\widetilde{f}$, $\widetilde{J}_{i}, \widetilde{\mathcal{M}}_{i}, i=1, \ldots, p$, satisfying conditions of Lemma 4.1 and such that

$$
|f(t, x, y)| \leq \widetilde{f}(t,|x|,|y|)
$$

for almost every $t \in[0, T]$ and all $x, y \in \mathbb{R}$, and

$$
\left|J_{i}(t, x)\right| \leq \widetilde{J}_{i}(|x|), \quad\left|\mathcal{M}_{i}(t, x)\right| \leq \widetilde{\mathcal{M}}_{i}(|x|)
$$


for $t \in[0, T], x \in \mathbb{R}, i=1, \ldots, p$. Finally assume that $C_{1}, C_{2}, L_{1}$ are constants from Lemma 4.1 and that (2.15), (2.16), (2.17) hold with $K$ and $K_{1}$ from Lemma 4.1. Then problem (2.1)-(2.3) has a solution $z$ such that

$$
\sup _{t \in[0, T]}|z(t)| \leq K, \quad \sup _{t \in[0, T]}\left|z^{\prime}(t)\right| \leq K_{1} .
$$

Proof. Consider the set $\mathcal{B}$ given by (2.18) with $K_{1}$ and $K$ from Lemma 4.1. It is obvious that Lemmas 2.2 and 2.3 are valid. Therefore if we introduce $\Omega$ by (3.1), we can define the operator $\mathcal{F}: \bar{\Omega} \rightarrow X$ by (3.3). Arguing as in the first part of the proof of Lemma 3.1, we get that $\mathcal{F}$ is compact on $\bar{\Omega}$.

Choose $\left(u_{1}, \ldots, u_{p+1}\right) \in \bar{\Omega}$. Then $\left\|u_{i}\right\|_{\infty} \leq K,\left\|u_{i}^{\prime}\right\|_{\infty} \leq K_{1}$ for $i=1, \ldots$, $p+1$. Using (4.6) and (4.7), we deduce from (3.4)

$$
\left|x_{j}^{\prime}(t)\right| \leq C_{1} \int_{0}^{T} \widetilde{f}\left(t, K, K_{1}\right) d t+L_{1}+C_{1} \sum_{i=1}^{p} \widetilde{\mathcal{M}}_{i}(K)+C_{2} \sum_{i=1}^{p} \widetilde{J}_{i}(K)
$$

for $t \in[0, T], j=1, \ldots, p+1$, and by virtue of (4.2) we get

$$
\left\|x_{j}^{\prime}\right\|_{\infty}<K_{1}, \quad j=1, \ldots, p+1 .
$$

Arguing as in the proof of Theorem 3.2, we deduce from (3.3) that

$$
x_{1}(0)-a x_{1}^{\prime}(0)=c_{1} .
$$

In addition, (4.8) yields

$$
\rho:=\max \left\{\left\|x_{j}^{\prime}\right\|_{\infty}: j=1, \ldots, p+1\right\}<K_{1} .
$$

Consequently, by (4.9) and (4.10),

$$
\left|x_{1}(t)\right| \leq(a+T) \rho+\left|c_{1}\right|, \quad t \in[0, T] .
$$

Further, (3.3) gives $x_{2}\left(\tau_{1}\right)=x_{1}\left(\tau_{1}\right)+J_{1}\left(\tau_{1}, u_{1}\left(\tau_{1}\right)\right)$, and, due to (4.7), (4.10) and (4.11),

$$
\left|x_{2}(t)\right| \leq(a+2 T) \rho+\left|c_{1}\right|+\widetilde{J}_{1}(K), \quad t \in[0, T] .
$$

Similarly we derive

$$
\left|x_{j+1}(t)\right| \leq(a+(j+1) T) \rho+\left|c_{1}\right|+\sum_{i=1}^{j} \widetilde{J}_{i}(K), \quad t \in[0, T], \quad j=1, \ldots, p .
$$

According to (4.3), (4.4), (4.10) and (4.12), we get

$$
\left\|x_{j}\right\|_{\infty} \leq K, \quad j=1, \ldots, p+1 .
$$

The estimates (4.8) and (4.13) imply that $\left(x_{1}, \ldots, x_{p+1}\right) \in \bar{\Omega}$. Consequently $\mathcal{F}(\bar{\Omega}) \subset \bar{\Omega}$ and the Schauder fixed point theorem yields a fixed point $\left(u_{1}, \ldots\right.$, $\left.u_{p+1}\right)$ in $\bar{\Omega}$. To get a solution $z$ of problem (2.1)-(2.3) we can repeat the proof of Theorem 3.2. 
ExAmple 4.3. Choose for simplicity $T=p=a=b=c_{1}=c_{2}=1$ and consider functions

$$
\left\{\begin{array}{l}
f(t, x, y)=\sin (4 t)(\sqrt[3]{x}+\sqrt[3]{y}), \quad J_{1}(t, x)=t \sqrt{|x|}, \\
\mathcal{M}_{1}(t, x)=t^{2} \sqrt[3]{x}, \quad t \in[0,1], x, y \in \mathbb{R} .
\end{array}\right.
$$

Then conditions (4.6) and (4.7) are satisfied for

$$
\left\{\begin{array}{l}
\tilde{f}(t, x, y)=|\sin (4 t)|(\sqrt[3]{|x|}+\sqrt[3]{|y|}), \quad \widetilde{J}_{1}(x)=\sqrt{|x|}, \\
\widetilde{\mathcal{M}}_{1}(x)=\sqrt[3]{|x|}, \quad t \in[0,1], x, y \in \mathbb{R} .
\end{array}\right.
$$

Since

$$
\lim _{x \rightarrow \infty} \frac{1}{x} \int_{0}^{1}|\sin (4 t)|(\sqrt[3]{|x|}+\sqrt[3]{|y|}) d t+\sqrt{|x|}+\sqrt[3]{|x|}=0,
$$

functions $\widetilde{f}, \widetilde{J}_{1}$ and $\widetilde{\mathcal{M}}_{1}$ fulfil (4.1). Further, the solution $\ell$ of problem (2.7), (2.2) has here the form $\ell(t) \equiv 1$ and $C_{0}=4 / 3, C_{1}=2 / 3, C_{2}=1 / 3$ are constants of (2.12). By Lemma 4.1, there exist $K_{1}>0$ and $K>K_{1}$ satisfying (4.2) and (4.3) with $\alpha=3$. Moreover from (4.3) we get that $K>1$ and consequently from (4.2) we obtain $K_{1}>1$. Let us put

$$
\gamma(x)=\frac{1}{K_{1}}\left(\frac{1}{2}-\frac{x^{2} \operatorname{sgn} x}{3 K^{2}}\right) \quad \text { for } x \in \mathbb{R} .
$$

Then

$$
\gamma^{\prime}(x)=-\frac{2|x|}{3 K^{2} K_{1}} \quad \text { for } x \in \mathbb{R},
$$

and we can easily check that $0<\gamma(x)<1,-1 / K_{1}<\gamma^{\prime}(x) \leq 0, J_{1}(t, x) \geq 0$ for $t \in[0,1], x \in[-K, K]$. Therefore (2.15), (2.16) and (2.17) are valid. Choose for example $K_{1}=5$. Then there exists $K \in(20.5,20.6)$ which is a solution of the equation

$$
K-\sqrt{K}-16=0,
$$

and which fulfils the inequality

$$
5>\frac{2}{3} \int_{0}^{1}|\sin (4 t)|(\sqrt[3]{K}+\sqrt[3]{5}) d t+\frac{2}{3} \sqrt[3]{K}+\frac{1}{3} \sqrt{K} .
$$

By Theorem 4.2, problem (2.1)-(2.3) with the data functions given by (4.14) and (4.15), where $K_{1}=5$ and $K \approx 20.531$ is a solution of equation (4.16), has a solution $z$ such that

$$
\sup _{t \in[0, T]}|z(t)|<20.6, \quad \sup _{t \in[0, T]}\left|z^{\prime}(t)\right| \leq 5 .
$$




\section{REFERENCES}

[1] I. BAJO AND E. Liz,Periodic boundary value problem for first order differential equations with impulses at variable times, J. Math. Anal. Appl. 204 (1996), 65-73.

[2] D.D. Bainov and V. Covachev, Impulsive Differential Equations With Small Parameter, in: Series on Advances in Mathematics for Applied Sciences, vol. 4, World Scientific, Singapore, 1994.

[3] D. Bainov and P. Simeonov, Impulsive Differential Equations: Periodic Solutions and Applications, Pitman Monographs and Surveys in Pure and Applied Mathematics 66, Longman Scientific and Technical, Essex 1993.

[4] G. Ballinger and X. Liu, Practical stability of impulsive delay differential equations and applications to control problems, Optimization Methods and Applications Appl. Optim., 52, Kluwer Academic, Dordrecht, (2001), pp. 3-21.

[5] J. Belley and M. Virgilio, Periodic Duffing delay equations with state dependent impulses, J. Math. Anal. Appl. 306 (2005), 646-662.

[6] _ Periodic Liénard-type delay equations with state-dependent impulses, Nonlinear Anal. 64 (2006), 568-589.

[7] M. Benchohra, J.R. Graef, S.K. Ntouyas and A. Ouahab, Upper and lower solutions method for impulsive differential inclusions with nonlinear boundary conditions and variable times, Dyn. Contin. Discreate Impuls. Syst. Ser, A Math. Anal. 12 (2005), 383-396.

[8] F. Cordova-Lepe, M. Pinto and E. Gonzalez-Olivares, A new class of differential equations with impulses at instants dependent on preceding pulses. Applications to management of renewable resources, Nonlinear Anal. Real World Appl. 13 (2012), 2313-2322.

[9] M. Frigon And D. O'Regan, First order impulsive initial and periodic problems with variable moments, J. Math. Anal. Appl. 233 (1999), 730-739.

[10] _ Second order Sturm-Liouville BVP's with impulses at variable times, Dyn. Contin. Discrete Impuls. Syst. Ser A: Math. Anal. 8 (2001), 149-159.

[11] J.J. Jiao, S.H. Cai ANd L.S. Chen, Analysis of a stage-structured predator-prey system with birth pulse and impulsive harvesting at different moments, Nonlinear Anal. Real World Appl. 12 (2011), 2232-2224.

[12] V. Lakshmikantham, D.D. Bainov and P.S. Simeonov, Theory of Impulsive Differential Equations, World Scientific, Singapore, 1989.

[13] X. Liu and G. Ballinger, Boundedness for impulsive delay differential equations and applications to population growth models, Nonlinear Anal. 53 (2003), no. 7-8, 1041-1062.

[14] L. Nie, Z. Teng, L. Hu AND J. Peng, Qualitative analysis of a modified Leslie-Gower and Holling - type II predator-prey model with state dependent impulsive effects, Nonlinear Anal. RWA 11 (2010), 1364-1373.

[15] L. Nie, Z. Teng And A. Torres, Dynamic analysis of an SIR epidemic model with state dependent pulse vaccination, Nonlinear Anal. Real World Appl. 13 (2012), 1621-1629.

[16] I. RachŮnková And J. TOMEČEk, Singular Dirichlet problem for ordinary differential equation with impulses, Nonlinear Anal. 65 (2006), 210-229.

[17] _ New approach to BVPs with state-dependent impulses, Bound. Value Probl. 22 (2013), 13 pp.

[18] _ Second order BVPs with state-dependent impulses via lower and upper functions, Cent. Eur. J. Math. 12 (1) (2014), 128-140.

[19] A.M. Samollenko and N.A. Perestyuk, Impulsive Differential Equations, World Scientific, Singapore, 1995. 
[20] I.M. Stamova AND G.T. Stamov, Lyapunov-Razumikhin method for impulsive functional differential equations and applications to the population dynamics, J. Comput. Appl. Math. 130 (2001), no. 1-2, 163-171.

[21] S. Tang and L. Chen, Density-dependent birth rate birth pulses and their population dynamic consequences, J. Math. Biol. 44 (2002), 185-199.

[22] F. WANG, G. PANG AND L. Chen, Qualitative analysis and applications of a kind of statedependent impulsive differential equations, J. Comput. Appl. Math. 216 (2008), 279-296.

[23] T. YAng, Impulsive Systems and Control: Theory and Applications, Nova Science Publishers Inc., 2001.

Manuscript received December 12, 2012

IRENA RAChŮNKOVÁ AND JAN TOMEČEK

Department of Mathematical Analysis

and Applications of Mathematics

Faculty of Science

Palacký University

17. listopadu 12

77146 Olomouc, CZECH REPUBLIC

E-mail address: irena.rachunkova@upol.cz, jan.tomecek@upol.cz 